\title{
Photonic bands of metallic systems. I. Principle of calculation and accuracy
}

\section{$\operatorname{AUTHOR}(\mathrm{S}):$}

Sakoda, K; Kawai, N; Ito, T; Chutinan, A; Noda, S; Mitsuyu, T; Hirao, K

\section{CITATION:}

Sakoda, K ...[et al]. Photonic bands of metallic systems. I. Principle of calculation and accuracy. PHYSICAL REVIEW B 2001, 64(4): 045116.

\section{ISSUE DATE:}

2001-07-15

URL:

http://hdl.handle.net/2433/39848

RIGHT:

Copyright 2001 American Physical Society 
PHYSICAL REVIEW B, VOLUME 64, 045116

\title{
Photonic bands of metallic systems. I. Principle of calculation and accuracy
}

\author{
Kazuaki Sakoda,* Noriko Kawai, and Takunori Ito \\ Research Institute for Electronic Science, Hokkaido University, North 12 West 6, Kita-ku, Sapporo 060-0812, Japan \\ Alongkarn Chutinan \\ Hirao Active Glass Project, Exploratory Research for Advanced Technology, Japan Science and Technology Corporation, \\ Keihanna Plaza, Seika-cho, Kyoto 619-0237, Japan \\ Susumu Noda \\ Department of Electronic Science and Engineering, Graduate School of Engineering, Kyoto University, Yoshida Honmachi, \\ Sakyo-ku, Kyoto 606-8501, Japan \\ Tsuneo Mitsuyu \\ Hirao Active Glass Project, Exploratory Research for Advanced Technology, Japan Science and Technology Corporation, \\ Keihanna Plaza, Seika-cho, Kyoto 619-0237, Japan \\ Kazuyuki Hirao \\ Department of Material Chemistry, Graduate School of Engineering, Kyoto University, Yoshida Honmachi, \\ Sakyo-ku, Kyoto 606-8501, Japan \\ (Received 2 February 2000; revised manuscript received 5 February 2001; published 9 July 2001)
}

\begin{abstract}
The dispersion relation, the field distribution, and the lifetime of the radiational eigenmodes in twodimensional photonic crystals composed of metallic cylinders were calculated for the $E$ polarization by means of the numerical simulation of the dipole radiation based on the finite-difference time-domain (FDTD) method. The convergence and the central processing unit time were compared with the plane-wave expansion method. The opaque frequency ranges in the transmission spectra calculated by the method of Pendry and MacKinnon corresponded quite well to the band gaps and the antisymmetric modes found in the photonic band diagram. The dispersion relation and the symmetry of the eigenmodes obtained by the numerical calculation were consistent with the prediction of the group theory and the analytical expression by the long-wavelength approximation.
\end{abstract}

DOI: 10.1103/PhysRevB.64.045116

PACS number(s): 78.66.Bz, 41.20.Jb, 02.60.Cb

\section{INTRODUCTION}

The dispersion relation and the density of states of the radiation field in photonic crystals are substantially different from those in free space. ${ }^{1-4}$ When their crystal structure is appropriately designed and the amount of the spatial modulation of their dielectric constant is large enough, frequency ranges called photonic band gaps can appear in which no electromagnetic eigenmode is allowed to exist. Optical properties of atoms and molecules embedded in such photonic crystals can also be quite different from those in free space or uniform materials. For example, if the transition frequency of the embedded atoms lies in the photonic band gap, no optical transition is allowed and spontaneous emission of photons is completely forbidden. In the very beginning of the extensive investigation of photonic crystals, Yablonovitch pointed out these types of drastic changes of the optical properties and their possible application to light-emitting devices. $^{5}$

In most theoretical investigations reported so far, the dielectric constants of the photonic crystals were assumed to be independent of frequency. When we deal with transparent materials whose optical transition frequencies are far from those of the relevant radiation field, this assumption is reasonable. However, when we deal with materials whose reso- nant polarization plays an important role in their optical response, we cannot neglect the frequency dependence of their dielectric constants.

To our knowledge, a small number of numerical works have been reported related to this problem. Kuzmiak and co-workers treated two-dimensional photonic crystals composed of metallic cylinders with a dielectric constant of the Drude type by means of the plane-wave expansion method. ${ }^{6-8}$ Nojima calculated the dispersion relation of exciton polaritons in a one-dimensional photonic crystal. ${ }^{9}$ The calculation was performed by searching the zero points of the determinant of the coefficients obtained by the planewave expansion. Yannopapas et al. reported the dispersion relation and transmission spectra of fcc lattices composed of metallic spheres. ${ }^{10}$ The calculation was performed by the transfer matrix method based on the vectorial KorringaKohn-Rostoker (KKR) formalism. ${ }^{11,12}$ In this case, spherical waves were used as a basis set, and good numerical accuracy was attained with a small number of spherical waves. The same holds for a two-dimensional array of circular cylinders, since we may use cylindrical waves as the basis set. ${ }^{13}$ However, in general cases in which we cannot assume a spherical or cylindrical symmetry for the components of the crystal, we have to apply a more general method such as the planewave expansion in spite of its slow convergence. 
Since spheres and cylinders are the simplest structures to be analyzed, they have been treated by the plane-wave expansion method as well. As Kuzmiak et al. reported for the two-dimensional array of metallic cylinders, in contrast with the frequency-independent case, the eigenvalue equation satisfied by the expansion coefficients is not linear with $\omega^{2} / c^{2}$, where $\omega$ is the angular frequency of the radiation field and $c$ is the light velocity in free space. So they had to linearize the eigenvalue equation by taking a larger basis set, which resulted in a heavier computational task. In the case of the $E$ polarization for which the electric field is parallel to the cylinder axis and perpendicular to the two-dimensional plane, the linearization procedure needed triple plane waves. In the case of the $H$ polarization for which the magnetic field is parallel to the cylinder axis, quadruple plane waves were necessary. Usually the central processing unit (CPU) time for the diagonalization of a matrix is proportional to the cube of its dimension, and hence to the cube of the number of the plane waves. So the computaional task was 27 times heavier for the $E$ polarization and 64 times heavier for the $H$ polarization than the frequency-independent case. Extension of this method to three-dimensional systems and more complicated systems such as those containing structural defects seems difficult, as a much larger number of plane waves are necessary for an accurate calculation.

One of the present authors and his collaborator reported another method based on the numerical simulation of the dipole radiation by means of the finite-difference timedomain (FDTD) method. ${ }^{14}$ Originally, this method was applied to the problem of point defects in a two-dimensional square crystal, and an excellent agreement between the theoretical calculation and the experimental observation was shown. ${ }^{15}$ Later, it was applied to point defects in a hexagonal crystal, ${ }^{16}$ line defects in a square crystal, ${ }^{17}$ and the evaluation of the quality factor in the presence of dielectric loss. ${ }^{18}$ In this method, the electromagnetic field radiated by an oscillating point dipole is calculated as a function of the oscillation frequency. The eigenfrequency is obtained as a resonance frequency, i.e., as a peak frequency of the radiation spectrum. The emitted field at the resonance frequency gives the eigenfunction. The CPU time for this method is essentially proportional to the number of the representative points on the spatial mesh used for the discretization of Maxwell's equations, which is in marked contrast to the case of the plane-wave expansion method for which the CPU time is proportional to the cube of the number of the plane waves. In addition, the FDTD algorithm is especially suitable for the vector processing and parallel computing, and so, the CPU time can be reduced greatly by using a computer with a vector processor or a parallel machine. Therefore, the numerical simulation of the dipole radiation is superior to the plane-wave expansion method when large and/or complicated systems are analyzed. For example, we can deal with a larger supercell than that usually assumed in the plane-wave expansion method when we calculate the eigenfrequencies and the eigenfunctions of localized defect modes. Thus the impurity-band effect can be eliminated. ${ }^{16}$ Three-dimensional systems can also betreated, as was shown by the excellent calculation by Hwang et al. ${ }^{19}$ The spatial symmetry of the eigenmodes is rigorously taken into account as well by imposing an appropriate boundary condition when we solve the difference equations derived from Maxwell's equations. The lifetime of the eigenmodes can be obtained by evaluating the temporal decrease in the accumulated radiation energy after switching off the oscillation of the dipole.

In this study, we analyzed the radiation field in twodimensional metallic systems. The present work consists of two papers. This paper (Paper I) deals mainly with the principle and accuracy of our numerical method. The convergence and the CPU time of our method will be compared with the plane-wave expansion method. The dispersion relation, the field distribution, and the lifetime of the radiational eigenmodes in two-dimensional square crystals composed of metallic cylinders calculated for the $E$ polarization will be presented and compared with the prediction of the group theory. The transmission spectra calculated by the method of Pendry and MacKinnon ${ }^{20}$ will be compared with the dispersion relation, and good correspondence between them will be shown. In the following paper (Paper II), ${ }^{21}$ we will deal with the $H$ polarization of the same system and show the presence of eigenmodes with extremely small group velocites due to the localized nature of surface plasmons. This feature will be further clarified by comparing their eigenfrequencies and eigenfunctions with the Mie resonance states for a single metallic cylinder.

\section{THEORY}

Let us explain our method here. We consider the radiation process of an oscillating dipole moment that is embedded in the photonic crystal. We begin with the following two equations (MKS units):

$$
\begin{gathered}
\boldsymbol{\nabla} \times \mathbf{E}(\mathbf{r}, t)=-\mu_{0} \frac{\partial}{\partial t} \mathbf{H}(\mathbf{r}, t), \\
\boldsymbol{\nabla} \times \mathbf{H}(\mathbf{r}, t)=\frac{\partial}{\partial t}\left\{\mathbf{D}_{0}(\mathbf{r}, t)+\mathbf{P}_{d}(\mathbf{r}, t)\right\},
\end{gathered}
$$

where $\mathbf{E}(\mathbf{r}, t), \mathbf{H}(\mathbf{r}, t)$, and $\mu_{0}$ denote the electric field, the magnetic field, and the magnetic permeability of free space, respectively. In Eq. (2), $\mathbf{P}_{d}(\mathbf{r}, t)$ stands for the oscillating dipole moment:

$$
\mathbf{P}_{d}(\mathbf{r}, t)=\boldsymbol{\mu} \delta\left(\mathbf{r}-\mathbf{r}_{0}\right) \exp (-i \omega t)
$$

where $\boldsymbol{\mu}$ and $\mathbf{r}_{0}$ are the magnitude and the position of the dipole moment, and $\delta$ is Dirac's delta function. $\mathbf{D}_{0}(\mathbf{r}, t)$ denotes the electric displacement due to the regular dielectric structure of the photonic crystal. It is generally given by the convolution integral of the electric field and the dielectric response function $\Phi(\mathbf{r}, t)$ :

$$
\mathbf{D}_{0}(\mathbf{r}, t)=\varepsilon_{0} \int_{-\infty}^{\infty} d t^{\prime} \Phi\left(\mathbf{r}, t-t^{\prime}\right) \mathbf{E}\left(\mathbf{r}, t^{\prime}\right),
$$


where $\varepsilon_{0}$ is the permittivity of free space. $\Phi(\mathbf{r}, t)$ is given by the Fourier transform of the dielectric constant $\varepsilon(\mathbf{r}, \omega)$, which is now a function of frequency as well as the spatial coordinates:

$$
\Phi(\mathbf{r}, t)=\frac{1}{2 \pi} \int_{-\infty}^{\infty} d \omega \varepsilon(\mathbf{r}, \omega) \exp (-i \omega t) .
$$

Note that $\Phi(\mathbf{r}, t)$ should satisfy the causality, i.e.,

$$
\Phi(\mathbf{r}, t)=0 \quad \text { for } \quad t<0,
$$

which implies that $\varepsilon(\mathbf{r}, \omega)$ does not have a pole in the upper half of the complex $\omega$ plane.

In order to treat the particular problem discussed by Kuzmiak et al. ${ }^{6-8}$ we consider a photonic crystal that contains metallic components and assume a dielectric constant of the Drude type in the metal:

$$
\varepsilon_{m}(\mathbf{r}, \omega)=\varepsilon_{\infty}\left[1-\frac{\omega_{p}^{2}}{(\omega+i \delta)(\omega+i \gamma)}\right],
$$

where $\varepsilon_{\infty}$ is the dielectric constant at sufficiently high frequencies, $\omega_{p}$ is the plasma frequency, $\gamma$ is the relaxation rate, and $\delta$ is a positive infinitesimal. In Eq. (7), we took into account the imaginary part of the dielectric constant in order to fulfill the Kramers-Kronig relation and hence the causality. Then Eq. (5) leads to

$$
\Phi(\mathbf{r}, t)=\varepsilon_{\infty} \delta(t)+\frac{\varepsilon_{\infty} \omega_{p}^{2}}{\gamma}[1-\exp (-\gamma t)] \theta(t),
$$

where $\theta(t)$ is a unit step function. From Eqs. (4) and (8), we obtain

$$
\frac{1}{\varepsilon_{0}} \frac{\partial}{\partial t} \mathbf{D}_{0}(\mathbf{r}, t)=\varepsilon_{\infty} \frac{\partial}{\partial t} \mathbf{E}(\mathbf{r}, t)+\varepsilon_{\infty} \omega_{p}^{2} \int_{0}^{\infty} d t^{\prime} e^{-\gamma t^{\prime}} \mathbf{E}\left(\mathbf{r}, t-t^{\prime}\right)
$$

in the metallic region. On the other hand, we assume that the dielectric constant is frequency independent outside the metal, i.e.,

$$
\varepsilon(\mathbf{r}, \omega)=\varepsilon_{b}(\mathbf{r}) .
$$

Thus we have

$$
\mathbf{D}_{0}(\mathbf{r}, t)=\varepsilon_{0} \varepsilon_{b}(\mathbf{r}) \mathbf{E}(\mathbf{r}, t) .
$$

In the FDTD calculation, we discretized Eqs. (1) and (2) to obtain difference equations, ${ }^{22}$ and solved the latter numerically with initial conditions $\mathbf{E}=\mathbf{0}$ and $\mathbf{H}=\mathbf{0}$, and boundary conditions

$$
\begin{aligned}
\mathbf{E}(\mathbf{r}+\mathbf{a}, t) & =\exp (i \mathbf{k} \cdot \mathbf{a}) \mathbf{E}(\mathbf{r}, t), \\
\mathbf{H}(\mathbf{r}+\mathbf{a}, t) & =\exp (i \mathbf{k} \cdot \mathbf{a}) \mathbf{H}(\mathbf{r}, t),
\end{aligned}
$$

where $\mathbf{k}$ is a wave vector in the first Brillouin zone and $\mathbf{a}$ is the elementary lattice vector. The latter conditions extract the contribution to the radiated electromagnetic field from particular eigenmodes with the specified wave vector. We can thus calculate the resonance frequency as a function of $\mathbf{k}$, i.e., we can obtain the dispersion relation. By the boundary conditions, Eqs. (12) and (13), it is enough to treat a unit cell in the numerical calculation, and we do not need additional boundary conditions such as the absorbing boundary condition, which is often necessary when we treat a finite volume by the FDTD calculation. ${ }^{22}$ This fact resulted in a small CPU time. The computation of the integral in the second term on the right-hand side of Eq. (9) may seem time-consuming at a first glance. But it is not, since the kernel has a simple form. When we denote the electric field at time $t=p \Delta t$ by $\mathbf{E}^{(p)}(\mathbf{r})$, where $\Delta t$ is the interval of the temporal mesh points and $p$ is an integer, the integral is approximated by

$$
\mathbf{F}^{(p)}(\mathbf{r})=\sum_{q=0}^{p} \Delta t e^{-q \gamma \Delta t} \mathbf{E}^{(p-q)}(\mathbf{r}) .
$$

$\mathbf{F}^{(p)}(\mathbf{r})$ can easily be calculated, since we have the following recursive equation:

$$
\mathbf{F}^{(p+1)}(\mathbf{r})=\Delta t \mathbf{E}^{(p+1)}(\mathbf{r})+e^{-\gamma \Delta t} \mathbf{F}^{(p)}(\mathbf{r})
$$

with $\mathbf{F}^{(0)}(\mathbf{r})=\mathbf{0}$. So, the increase in the numerical task compared with the case of frequency-independent dielectric constants is not serious, and it is actually several tens of percent.

In the next section, we will first present the band structure of a three-dimensional simple cubic lattice composed of dielectric spheres with a frequency-independent dielectric constant and compare the convergence and the CPU time with the case of the plane-wave expansion method. Here we dealt with the frequency-independent case since the plane-wave expansion method is impractical for the frequency-dependent case. Next we will present the band structure and the field distribution of the two-dimensional square lattice composed of metallic cylinders for the $E$ polarization. We will show that the eigenmodes for the $E$ polarization have their replicas in free space, and the group-theoretical assignment of their symmetries based on the reduction of the reducible representations given by the linear combination of plane waves in free space is satisfactory. We will also show that the longwavelength approximation gives an appropriate description of the band structure of the metallic system in the lowfrequency region.

Let us conclude this section by giving three remarks. First, our method is not restricted to dielectric constants of the Drude type. If we would like to treat semiconductors, for example, we may assume the following dielectric constant:

$$
\varepsilon_{s}(\omega)=\varepsilon_{\infty} \frac{\omega_{L}^{2}-\omega^{2}-i \omega \gamma}{\omega_{T}^{2}-\omega^{2}-i \omega \gamma},
$$

where $\omega_{T}\left(\omega_{L}\right)$ is the transverse (longitudinal) exciton frequency and $\gamma$ is the relaxation rate. In this case, the response function is given as follows:

$$
\begin{aligned}
\Phi(t)= & \varepsilon_{\infty} \delta(t)+i \varepsilon_{\infty} \frac{\omega_{L}^{2}-\omega_{T}^{2}}{2 \Omega_{T}} \\
& \times\left\{e^{-\left(\Gamma+i \Omega_{T}\right) t}-e^{-\left(\Gamma-i \Omega_{T}\right) t}\right\} \theta(t),
\end{aligned}
$$

where 


$$
\Gamma=\frac{\gamma}{2}
$$

and

$$
\Omega_{T}=\sqrt{\omega_{T}^{2}-\frac{\gamma^{2}}{4}}
$$

We can deal with other cases as long as the assumed frequency-dependent dielectric constant satisfies the causality or the Kramers-Kronig relation.

Second, if the $\mathbf{k}$ vector in the first Brillouin zone chosen for the photonic band calculation has a certain symmetry, i.e., if the eigenmode for $\mathbf{k}$ should have a certain spatial symmetry expected by the group theory, we may impose an additional boundary condition on the electromagnetic field, which reduces the spatial region dealt with in the numerical calculation considerably and leads to the decrease in the CPU time.

Third, the present method can be extended to the case that the analytical form of the frequency-dependent dielectric constant is not known. This is a very important and practical feature of the present method. For example, let us assume that we know the values of the complex dielectric constant at certain frequencies by experimental observations. Thus we have a certain number of the pairs of the frequency and the complex dielectric constant, $\left\{\omega_{j}, \varepsilon_{j}\right\}$. The key idea is that the use of the response function given in Eq. (4) is necessary to describe the non-steady-state of the radiation field after the abrupt introduction of the oscillating dipole at $t=0$ and that the radiation field after a long period that can be regarded as in a steady state is well described by the dielectric constant at the oscillation frequency alone. This implies that the choice of the analytical form of the dielectric constant is not important when we calculate the radiation field at a given angular frequency $\omega_{j}$ and a given dielectric constant $\varepsilon_{j}$. For example, by choosing $\omega_{T}, \omega_{L}, \varepsilon_{\infty}$, and $\gamma$ appropriately for each $\omega_{j}$ so that $\varepsilon_{s}\left(\omega_{j}\right)=\varepsilon_{j}$, the radiation field in the realistic system, which is not described by an analytical expression of the dielectric constant, can be calculated by the present method.

As a demonstration of this remarkable feature, we calculated the eigenfrequencies of the metallic system described above by using the dielectric constant of a semiconductor given in Eq. (16). For $\omega_{j}<\omega_{p}$, we denote

$$
\varepsilon_{m}\left(\omega_{j}\right)=\varepsilon_{j}=\varepsilon_{j}^{\prime}+i \varepsilon_{j}^{\prime \prime} .
$$

We took

$$
\omega_{T}=\frac{\omega_{j}}{\sqrt{2}} \quad \text { and } \quad \omega_{L}=\sqrt{\frac{3}{2}} \omega_{j} .
$$

From Eq. (16), we have

$$
\gamma=\frac{\left(\varepsilon_{j}^{\prime}+\left|\varepsilon_{j}\right|\right) \omega_{T}}{\sqrt{2} \varepsilon_{j}^{\prime \prime}},
$$

TABLE I. Comparison between the eigen-angular-frequencies of the lowest symmetric band on the $\Delta$ point calculated with the metallic response function $(M)$, Eq. (8), and with that of the semiconductor $(S)$, Eq. (17). The wave number $k$ and the angular frequency $\omega$ are normalized with the lattice constant $a$ and the light velocity $c$. See text for details.

\begin{tabular}{ccc}
\hline \hline$k a / 2 \pi$ & $\omega a / 2 \pi c(M)$ & $\omega a / 2 \pi c(S)$ \\
\hline 0.1 & 0.750 & 0.752 \\
0.2 & 0.764 & 0.766 \\
0.3 & 0.785 & 0.786 \\
0.4 & 0.805 & 0.805 \\
0.5 & 0.814 & 0.815 \\
\hline \hline
\end{tabular}

$$
\varepsilon_{\infty}=\varepsilon_{j} \frac{2 \gamma^{2}+\omega_{T}^{2}}{2 \gamma^{2}-\omega_{T}^{2}}
$$

By substituting these values in Eq. (17) for each $\omega_{j}$, we calculated the spectra of the dipole radiation and obtained the resonance frequencies. Some results are listed in Table I where the eigenfrequencies obtained by the metallic and the semiconductorlike dielectric constants are compared. As is clearly seen, the difference is amazingly small and less than $0.3 \%$ for this example. This is apparent evidence for the fact that we can extend our method to the case in which the analytical form of the dielectric constant is not known.

\section{RESULTS AND DISCUSSION}

Figure 1 shows the dispersion relation of the simple cubic lattice composed of a dielectric sphere with the dielectric constant of 13 at each lattice point. The ordinate denotes the normalized eigenfrequency, where $a$ and $c$ denote the lattice constant and the light velocity in free space, respectively. The abscissa denotes the wave vector between the $\Gamma$ point, $(0,0,0)$, and $X$ point, $(\pi / a, 0,0)$, in the first Brillouin zone. The symmmetry of the magnetic field of each band is also shown, which is an irreducible representation of the $C_{4 v}$ point group. The unit cell was divided into $80 \times 80 \times 80$ parts and one period of the oscillation was divided into 320 steps in order to discretize Maxwell's equations. The further decrease of the size of the spatial and temporal meshes did not bring about an apparent change in the resonance frequencies as will be shown later. The symmetries shown in this figure were obtained by examining the field distributions of the eigenmodes. They are consistent with the prediction of the group theory based on the reduction of the reducible representations given by the linear combination of plane waves in free space, which was described in detail in Ref. 23.

Figure 2 shows the convergence properties for the FDTD and the plane-wave expansion methods, ${ }^{24}$ where the abscissa denotes the cubic root of the number of the spatial mesh, $\sqrt[3]{N}$, for the former and that of the basis plane waves, $\sqrt[3]{M}$, for the latter. The eigenfrequencies were evaluated at the middle point between the $\Gamma$ and $X$ points. The eigenfrequencies obtained by the FDTD method are represented by solid circles, whereas those obtained by the plane-wave expansion 


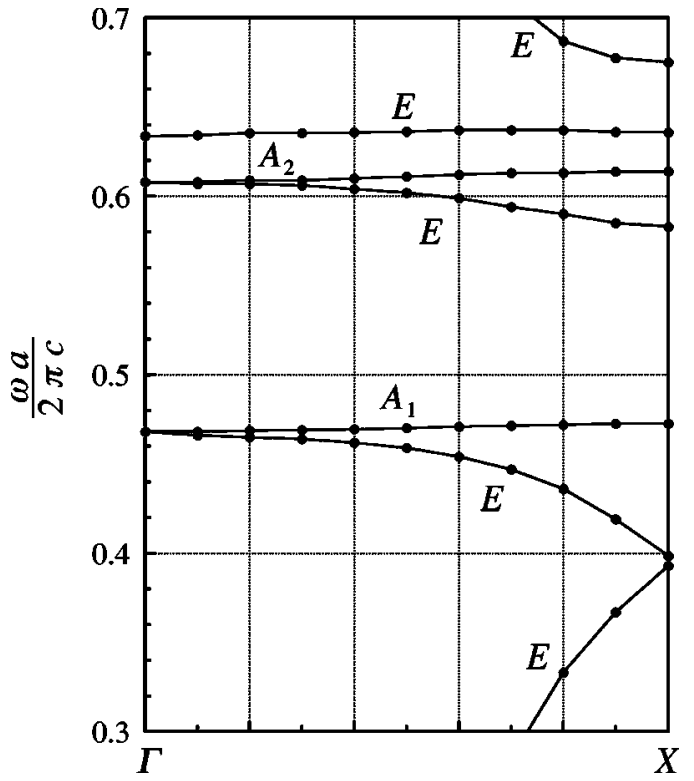

FIG. 1. The dispersion relation for the three-dimensional simple cubic lattice composed of a dielectric sphere at each lattice point. The ordinate denotes the normalized frequency where $a$ and $c$ stand for the lattice constant and the light velocity in free space, respectively. The abscissa denotes the wave vector, $\mathbf{k}$. The dispersion relation was calculated for $\mathbf{k}$ between the $\Gamma$ point, $(0,0,0)$, and the $X$ point, $(\pi / a, 0,0)$, in the first Brillouin zone. The symmetry of each band for the magnetic field is also shown, which is an irreducible representation of the $C_{4 v}$ point group. The following parameters were assumed for the numerical calculation: the dielectric constant of the spheres is 13.0, that of the background is 1.0; the ratio of the radius of the sphere to the lattice constant is $0.3: 1.0$.

method are represented by open circles. As we will see later, the plane-wave expansion method is impractical when $M$ is, say, greater than 4000 . Thus we plotted data obtained with $M \leqslant 4096$ in Fig. 2. It is apparent that the convergence at $\omega a / 2 \pi c>0.4$ is not satisfactory for the plane-wave expansion method even when $M=4096$. On the other hand, the CPU time for the FDTD method is relatively small and the

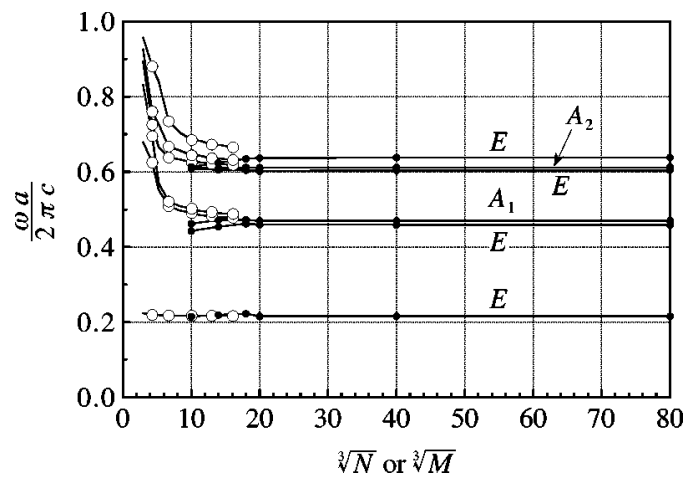

FIG. 2. The convergence behavior of the FDTD method (solid circles) and the plane-wave expansion method (open circles). The ordinate is the normalized frequency. The abscissa denotes the cubic root of the number of mesh points $N$ or that of the plane waves $M$. The same parameters as for Fig. 1 were used for numerical calculation.

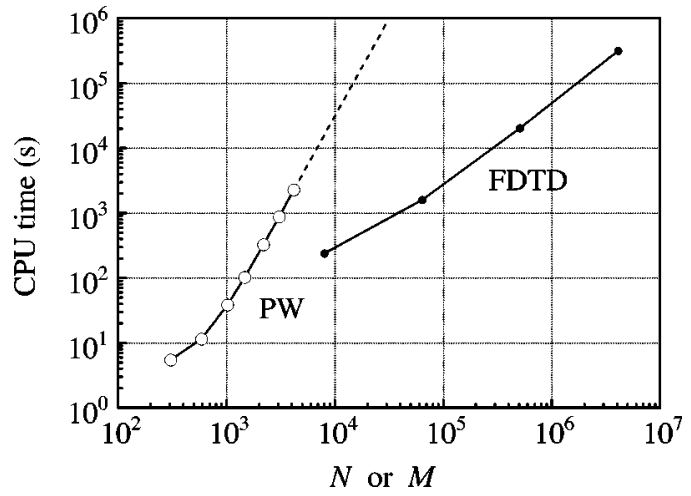

FIG. 3. The CPU time necessary for the numerical calculation for one wave vector in the first Brillouin zone as a function of the number of the mesh points $N$ or the number of the plane waves $M$. The supercomputer (Hitachi SR 8000) in Hokkaido University Computing Center was used. In the numerical calculation, one node that consisted of eight CPU's was used with the FORTRAN 90 compiler that enabled parallel computing in the node.

eigenfrequencies can be calculated with very large $N$. The convergence is really satisfactory as shown in Fig. 2. The CPU time for both methods is compared in Fig. 3. Since the CPU time depends on the source program, the compiler, the architecture of the computer, and so on, this figure should be considered to just give an estimation of the CPU time. However, it is apparent that the FDTD method is much superior than the plane-wave expansion method when we need accurate calculation with large $N$ or $M$.

Next let us present the results for the metallic systems. According to Kuzmiak et al., ${ }^{6-8}$ we analyzed twodimensional photonic crystals composed of a square array of metallic cylinders with a radius $r_{m}$. The following parameters were assumed: $r_{m} / a=0.472$ (crystal 1) or 0.0564 (crystal 2), $\varepsilon_{\infty}=1.0, \omega_{p} a / 2 \pi c=1.0$, and $\gamma=0.01 \omega_{p}$, where $a$ denotes the lattice constant. We will restrict our discussion as usual to the case that the wave vector $\mathbf{k}$ lies in the twodimensional $(x-y)$ plane. In this case, Maxwell's equations are decoupled into two polarization components. One is the $E$ polarization for which the electric field is perpendicular to the $x-y$ plane and the other is the $H$ polarization for which the magnetic field is perpendicular to the $x-y$ plane. We treat the $E$ polarization in this Paper. The $H$ polarization will be treated in Paper II.

In the actual calculation, we discretized the wave equation for $E_{z}$ that was obtained from Eqs. (1) and (2) by eliminating $H_{x}$ and $H_{y}$ and solved it. This simplified treatment for the $E$ polarization did not reduce the accuracy of the numerical calculation, since the wave equation for $E_{z}$ is not very singular due to the fact that $E_{z}$ and its derivatives of the first order are continuous. The two-dimensional unit cell was divided into $40 \times 40$ parts for crystal 1 and $120 \times 120$ parts for crystal 2 , and one period of the oscillation was divided into 160 steps for crystal 1 and 480 steps for crystal 2 in order to discretize the wave equation. The further decrease in the size of the spatial and temporal meshes did not bring about an apparent change in the resonance frequencies. The lifetime of the eigenmodes was evaluated by observing the temporal 


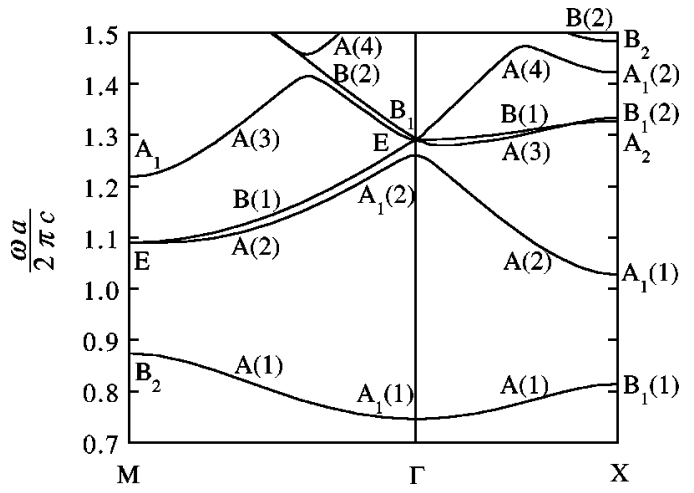

FIG. 4. The dispersion relation of the two-dimensional square photonic crystal composed of metallic cylinders (crystal 1) for the $E$ polarization calculated by means of the numerical simulation of the dipole radiation. The ordinate is the normalized frequency where $\omega$, $a$, and $c$ stand for the angular frequency of the radiation field, the lattice constant of the crystal, and the light velocity in free space. According to the previous calculation by Kuzmiak et al. (Refs. $6-8)$, the following parameters were used for numerical calculation: $r_{m} / a=0.472(f=0.7)$, where $r_{m}$ denotes the radius of the metallic cylinders, $\varepsilon_{\infty}=1.0, \omega_{p} a / 2 \pi c=1.0$, and $\gamma=0.01 \omega_{p}$ in Eq. (7). The dispersion relation was drawn for highly symmetric points in the first Brillouin zone of the two-dimensional crystal: $\Gamma(0,0)$, $X(\pi / a, 0)$, and $M(\pi / a, \pi / a)$. The spatial symmetry of each eigenmode for $E_{z}$ is also shown in this figure, where the number in parentheses is given in order of the ascending frequency when there is more than one mode of the same symmetry in the analyzed frequency region.

decrease in the accumulated electromagnetic energy after 200 cycles of the oscillation of the dipole moment. The total electromagnetic energy after switching off the oscillation showed an exponential decay and we could obtain the lifetime easily.

The dispersion relation for crystal 1 thus obtained is presented in Fig. 4 where the symmetry of each eigenmode is also shown. In this figure, the number in parentheses is given in order of the ascending frequency when there is more than one mode of the same symmetry in the analyzed spectral region. The symmetry assignments are consistent with the prediction of the group theory that was obtained by the comparison with the irreducible representations of the radiation field in free space. ${ }^{25}$ This fact implies that the radiational eigenmodes for the $E$ polarization in this frequency range are essentially modified plane waves. Note that there is no eigenmode for $\omega a / 2 \pi c<0.745$. We can show that this cutoff frequency is consistent with the long-wavelength approximation of Maxwell's equations. In this approximation, the dispersion relation is given by

$$
\frac{\omega^{2}}{c^{2}}=\frac{k^{2}}{\bar{\varepsilon}},
$$

where $\bar{\varepsilon}$ denotes the spatial average of the dielectric constant and is given by

$$
\bar{\varepsilon} \sim 1+\left(\varepsilon_{\infty}-1\right) f-\frac{f \varepsilon_{\infty} \omega_{p}^{2}}{\omega^{2}} .
$$
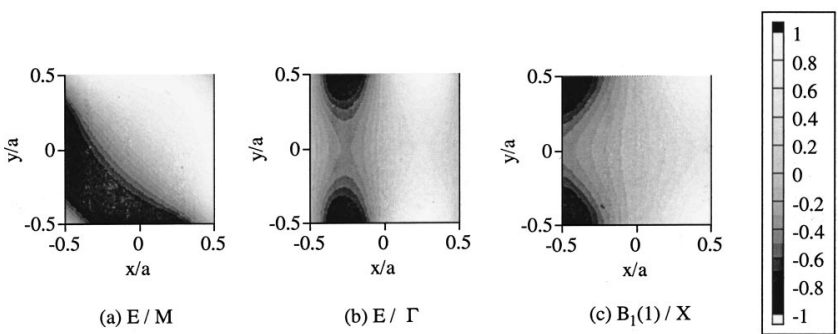

FIG. 5. The distribution of the electric field of (a) the $E$ mode at the $M$ point, (b) the $E$ mode at the $\Gamma$ point, and (c) the $B_{1}(1)$ mode at the $X$ point. The maximum of each electric field is normalized to unity. For the doubly degenerate $E$ modes, only one eigenfunction is shown. The other eigenfunction can be obtained by a rotation by $90^{\circ}$. For all eigenmodes, the eigenfunctions show their peculiar symmetries.

In this equation, $f$ is the filling factor of the metallic cylinders: $f=0.7$ for crystal 1 and $f=0.01$ for crystal 2 . From Eqs. (24) and (25), we obtain

$$
\omega=\sqrt{\frac{c^{2} k^{2}+f \varepsilon_{\infty} \omega_{p}^{2}}{1+\left(\varepsilon_{\infty}-1\right) f} .}
$$

Substituting the assumed parameters for crystal 1 into this equation, the cutoff frequency is obtained as 0.84 . This value is fairly close to the numerical result. Equation (26) also tells us that the eigenfrequency takes the smallest value at the $\Gamma$ point and increases parabolically with small $k$.

Figure 5 shows three typical examples of the eigenfunctions, that is, (a) the $E$ mode at the $M$ point, (b) the $E$ mode at the $\Gamma$ point, and (c) the $B_{1}(1)$ mode at the $X$ point. The maximum of each electric field is normalized to unity. Note that these eigenfunctions have the correct symmetries. Kuzmiak et al. reported a very curious observation for the case of $f=0.1$ in that these modes had exactly zero amplitudes. They concluded that these modes were some artifacts of the calculation and were not of real existence. ${ }^{8}$ This assertion is, however, completely wrong, as is evident from Fig. 5. The appearance of these modes is natural and consistent with the group theory since the $E$ mode at the $M$ point, for example, connects with the $A$ (symmetric) and $B$ (antisymmetric) modes on the $\Sigma$ point, which is expected from the compatibility relation. ${ }^{25}$

Next, Figs. 6 and 7 show the transmission spectra of crystal 1 with eight lattice layers calculated by the PendryMacKinnon method. ${ }^{20}$ The incident plane wave was assumed to be propagated in the $(1,0)(\Gamma-X)$ direction and the $(1,1)$ $(\Gamma-M)$ direction, respectively. In Table II, the opaque frequency ranges where the transmittance is less than 0.1 are compared with the band gaps and the frequency ranges where only antisymmetric $(B)$ modes exist. Note that the antisymmetric modes do not couple to the plane wave coming from outside of the photonic crystal at normal incidence because of the mismatching of the spatial symmetry, and they do not contribute to the transmittance. ${ }^{25,26}$ So, we refer to them as uncoupled modes. Figure 8 shows the lifetime of the five lowest eigenmodes. It depends strongly on the amount of the field distribution in the metallic region. 


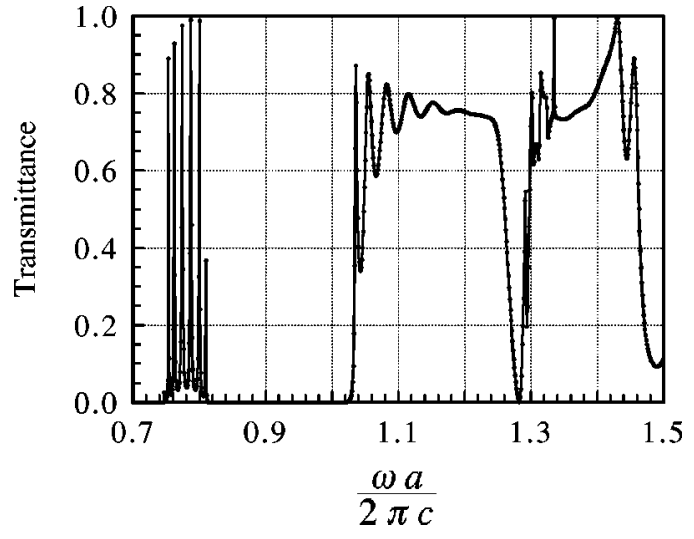

FIG. 6. The transmission spectrum in the $(1,0)$ direction calculated by the Pendry-MacKinnon method. The abscissa denotes the normalized frequency. The same parameters as for Fig. 4 were used for numerical calculation except that $\gamma$ was set to be zero. The number of the lattice layers was assumed to be eight.

The dispersion relation and the lifetime of the lowest band for crystal 2 with a filling factor of 0.01 are shown in Figs. 9 and 10. In the long-wavelength approximation, the cutoff frequency is given by $\omega a / 2 \pi c=0.1$, which is very close to the numerical result, 0.097 . Figure 10 shows a very interesting behavior of the lifetime of the lowest band. It varies more than three orders of magnitude with $\mathbf{k}$. This feature originates from the variation of the field distribution. For example, the $B_{2}$ mode on the $M$ point is antisymmetric about the $x$ and $y$ axes. Its amplitude is thus equal to zero on these axes. Because the electric field is continuous and the radius of the metallic cylinders is small for crystal 2, the electric field is small everywhere in the metal. This is the reason why the dielectric loss is small and the lifetime is long for the $B_{2}$ mode. On the other hand, the $A_{1}$ mode on the $\Gamma$ point is totally symmetric and it may have a large amplitude in the metallic region. This is the reason why its lifetime is much shorter than that of the $B_{2}$ mode.

Let us conclude this section by giving one remark. The dispersion relation shown in Fig. 4 is similar to that obtained

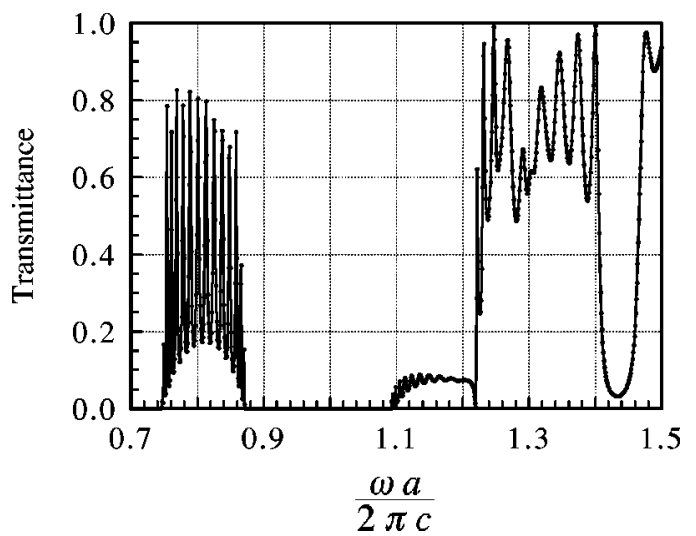

FIG. 7. The transmission spectrum in the $(1,1)$ direction calculated by the Pendry-MacKinnon method. The abscissa denotes the normalized frequency. The same parameters as for Fig. 6 were used for numerical calculation.
TABLE II. Comparison between the band structure and the transmission spectra.

\begin{tabular}{lccc}
\hline \hline Direction & Band gap & $B$ mode & Opaque range \\
\hline$\Gamma-X$ & $0.814-1.028$ & & $0.811-1.032$ \\
& $1.260-1.280$ & & $1.275-1.286$ \\
& 1.474 & & 1.483 \\
$\Gamma-M$ & $0.873-1.090$ & & $0.871-1.093$ \\
& & $1.415-1.458$ & $1.415-1.454$ \\
Cutoff & 0.745 & & 0.752 \\
\hline \hline
\end{tabular}

by the plane-wave expansion method, ${ }^{6}$ since the radiational eigenmodes for the $E$ polarization are essentially modified plane waves as we mentioned previously and their description by the linear combination of plane waves was a good approximation. However, this feature cannot be expected in more general cases. As a matter of fact, the $H$ polarization of the two-dimensional metallic system is a typical example, for which the localized nature of surface plasmons brings about a completely different feature that is difficult to deal with by the plane-wave expansion. This problem will be treated in Paper II. ${ }^{21}$

\section{CONCLUSION}

The dispersion relation, the field distribution, and the lifetime of the radiational eigenmodes in the two-dimensional photonic crystals composed of metallic cylinders were calculated by means of the numerical simulation of the dipole radiation based on the FDTD method. The CPU time for this method is proportional to the number of the representative points on the spatial mesh used for the discretization of Maxwell's equations, which is in marked contrast to the case of the plane-wave expansion method for which the CPU time is proportional to the cube of the number of the plane waves. So, the present method is superior to the plane-wave expansion method when large and/or complicated systems are analyzed. This feature was demonstrated by the photonic band calculation for a simple cubic crystal composed of dielectric spheres.

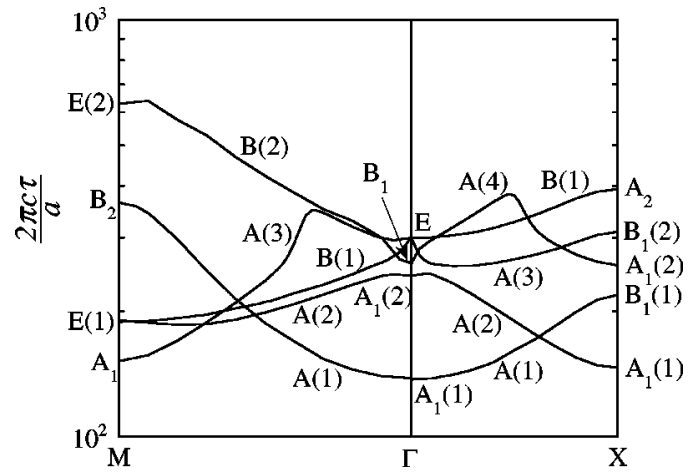

FIG. 8. The lifetime of the lowest five eigenmodes of crystal 1 . The same parameters as for Fig. 4 were used for numerical calculation. The ordinate denotes the normalized lifetime in a logarithmic scale. 


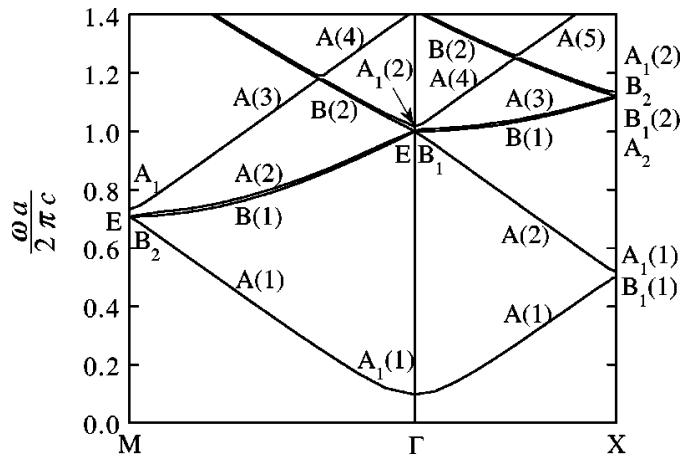

FIG. 9. The dispersion relation of the two-dimensional square photonic crystal composed of metallic cylinders (crystal 2) for the $E$ polarization calculated by means of the numerical simulation of the dipole radiation. The same parameters as for Fig. 4 were used for numerical calculation except that $r_{m} / a=0.0564(f=0.01)$.

The dispersion relation for the $E$ polarization obtained by the present work was similar to that reported previously by Kuzmiak et al. The symmetry of the eigenmodes was consistent with the prediction from group theory considerations that was obtained by the reduction procedure starting from the plane-wave representation of the unperturbed wave functions, which implies that the eigenmodes for the $E$ polarization are essentially modified plane waves. The opaque frequency ranges in the transmission spectra calculated by the Pendry-MacKinnon method corresponded quite well to the band gaps and the frequency ranges of the antisymmetric $(B)$ modes. The cutoff frequencies coincided with the longwavelength approximation of Maxwell's equations as well.

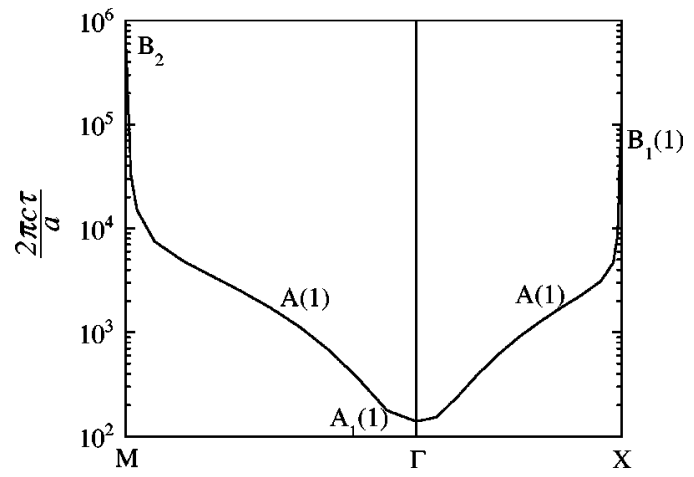

FIG. 10. The lifetime of the lowest eigenmode of crystal 2. The same parameters as for Fig. 9 were used for numerical calculation. The ordinate denotes the normalized lifetime in a logarithmic scale.

The curious observation reported by Kuzmiak et al. that some eigenmodes had zero amplitudes was denied by examining the field distribution of the related eigenmodes. We found that the lifetime of the eigenmodes depends strongly on the field distribution, and its variation of more than three orders of magnitude was observed.

\section{ACKNOWLEDGMENTS}

This work was supported by ERATO-JST (Exploratory Research for Advanced Technology of Japan Science and Technology Corporation), the Mitsubishi Foundation, and the Grant-in-Aid for Scientific Research on Priority Area "Photonic Crystals" from the Ministry of Education, Science, Sports, and Culture.
*Electronic address: sakoda@es.hokudai.ac.jp

†Present address: Department of Electronic Science and Engineering, Graduate School of Engineering, Kyoto University, Yoshida Honmachi, Sakyo-ku, Kyoto 606-8501, Japan.

†Present address: Advanced Technology Research Laboratories, Matsushita Electric Industrial Co., 3-4 Hikaridai, Seika-cho, Kyoto 619-0237, Japan.

${ }^{1}$ J.D. Joannopoulos, R.D. Meade, and J.N. Winn, Photonic Crystals (Princeton University Press, Princeton, 1995).

${ }^{2}$ Photonic Band Gaps and Localization, edited by C. M. Soukoulis (Plenum, New York, 1993).

${ }^{3}$ Photonic Band Gap Materials, edited by C. M. Soukoulis (Kluwer, Dordrecht, 1996).

${ }^{4}$ K. Sakoda, in Optical Properties of Low-Dimensional Materials, edited by T. Ogawa and Y. Kanemitsu (World Scientific, Singapore, 1998), Vol. 2, pp. 402-450.

${ }^{5}$ E. Yablonovitch, Phys. Rev. Lett. 58, 2059 (1987).

${ }^{6}$ V. Kuzmiak, A.A. Maradudin, and F. Pincemin, Phys. Rev. B 50, 16835 (1994).

${ }^{7}$ V. Kuzmiak and A.A. Maradudin, Phys. Rev. B 55, 7427 (1997).

${ }^{8}$ V. Kuzmiak and A.A. Maradudin, Phys. Rev. B 58, 7230 (1998).

${ }^{9}$ S. Nojima, Phys. Rev. B 57, R2057 (1998).

${ }^{10}$ V. Yannopapas, A. Modinos, and N. Stefanou, Phys. Rev. B 60, 5359 (1999).
${ }^{11}$ N. Stefanou, V. Karathanos, and A. Modinos, J. Phys.: Condens. Matter 4, 7389 (1992).

${ }^{12}$ K. Ohtaka and Y. Tanabe, J. Phys. Soc. Jpn. 65, 2276 (1996).

${ }^{13}$ K. Ohtaka, T. Ueta, and K. Amemiya, Phys. Rev. B 57, 2550 (1998).

${ }^{14}$ K. Sakoda and J. Kawamata, Opt. Express 3, 12 (1998).

${ }^{15}$ K. Sakoda and H. Shiroma, Phys. Rev. B 56, 4830 (1997).

${ }^{16}$ K. Sakoda, J. Appl. Phys. 84, 1210 (1998).

${ }^{17}$ K. Sakoda, T. Ueta, and K. Ohtaka, Phys. Rev. B 56, 14905 (1997).

${ }^{18}$ T. Ueta, K. Ohtaka, N. Kawai, and K. Sakoda, J. Appl. Phys. 84, 6299 (1998).

${ }^{19}$ J-K. Hwang, H-Y. Ryu, and Y-H. Lee, Phys. Rev. B 60, 4688 (1999).

${ }^{20}$ J.B. Pendry and A. MacKinnon, Phys. Rev. Lett. 69, 2772 (1992).

${ }^{21}$ T. Ito and K. Sakoda, following paper, Phys. Rev. B 64, 045117 (2001).

${ }^{22}$ A. Taflove, Computational Electrodynamics (Artech House, Boston, 1995).

${ }^{23}$ K. Sakoda, Phys. Rev. B 55, 15345 (1997).

${ }^{24}$ K.M. Ho, C.T. Chan, and C.M. Soukoulis, Phys. Rev. Lett. 65, 3152 (1990)

${ }^{25}$ K. Sakoda, Phys. Rev. B 52, 7982 (1995).

${ }^{26}$ W.M. Robertson, G. Arjavalingam, R.D. Meade, K.D. Brommer, A.M. Rappe, and J.D. Joannopoulos, Phys. Rev. Lett. 68, 2023 (1992). 